\title{
Prótesis total de codo en el contexto de una seudoartrosis de olécranon. Técnica quirúrgica y reporte de tres casos
}

Gerardo Gallucci, Ignacio Rellán, Jorge G. Boretto, Agustín Donndorff, Ezequiel E. Zaidenberg, Pablo De Carli Sector de Cirugía de Mano y Miembro Superior, Instituto de Ortopedia y Traumatología "Prof. Dr. Carlos E. Ottolenghi", Hospital Italiano de Buenos Aires, Ciudad Autónoma de Buenos Aires, Argentina

\section{RESUMEN}

La indemnidad del aparato extensor es fundamental para un correcto funcionamiento de una prótesis de codo. Se ha considerado que la deficiencia del tríceps es una contraindicación relativa para la artroplastia, porque produce una contractura en flexión y un déficit de extensión activa. Estas limitaciones pueden afectar significativamente la mejora funcional que la artroplastia total de codo produce. Ante una seudoartrosis de olécranon, la colocación de una prótesis total de codo se presenta como un problema complejo que resolver. El objetivo de este artículo es describir la técnica quirúrgica para la colocación de una prótesis total de codo en el contexto de una seudoartrosis de olécranon, y comunicar tres casos.

Palabras clave: Prótesis total de codo; seudoartrosis de olécranon; deficiencia del tríceps; sistema de absorbe-tracción.

Nivel de Evidencia: IV

\section{Total Elbow Arthroplasty in the Context of a Nounion of the Olecranon. Surgical Technique and Report} of 3 Cases

\section{ABSTRACT}

The integrity of the extensor apparatus is essential for the correct functioning of an elbow prosthesis. Triceps deficiency has been considered a relative contraindication for arthroplasty, because it produces a flexion contracture and an active extension deficit. These limitations can significantly affect the functional improvement that total elbow arthroplasty produces. Faced with an olecranon nonunion, the placement of a total elbow prosthesis is presented as a complex problem to be solved. The objective of this article is to describe the surgical technique for the placement of a total elbow prosthesis in the context of an olecranon nonunion, and to report three cases.

Keywords: Total elbow prosthesis; olecranon nonunion; triceps deficiency; absorber-traction system.

Level of Evidence: IV

\section{INTRODUCCIÓN}

La prótesis total de codo (PTC) es una opción eficaz para el tratamiento de diversas enfermedades postraumáticas y degenerativas. ${ }^{1-8} \mathrm{La}$ indemnidad del aparato extensor es fundamental para un correcto funcionamiento de la prótesis. ${ }^{9}$ Se ha considerado a la deficiencia del tríceps como una contraindicación relativa para la PTC, porque produce una contractura en flexión y un déficit de extensión activa. Estas limitaciones pueden afectar significativamente la mejora funcional que la artroplastia total de codo produce..$^{10,11}$ 
De esta manera, si un paciente tiene artrosis o artritis de codo asociada a una seudoartrosis de olécranon (SO), la colocación de una PTC como procedimiento de salvataje, se presenta como un problema complejo que resolver.

Si bien se han descrito algunas alternativas para el manejo de una SO en ese contexto, la bibliografía sigue siendo escasa. Algunos de los tratamientos propuestos incluyen la resección del extremo proximal del cúbito, la osteosíntesis y el tratamiento conservador en el caso de fracturas sin gran desplazamiento y con fuertes uniones fibrosas. ${ }^{12,13}$

El objetivo de este artículo es describir la técnica quirúrgica para la colocación de una PTC en el contexto de una SO y comunicar tres casos.

\section{Técnica quirúrgica}

Todos los pacientes fueron operados por el mismo cirujano. La cirugía se llevó a cabo con el paciente en decúbito dorsal con anestesia regional. Luego de colocar un manguito neumático, se realizó un abordaje posterior de codo y se identificó el nervio cubital que, luego de su neurólisis, fue transpuesto anteriormente en todos los casos. Se identificó el foco de la SO (Figura 1A) y, a través de este, se ingresó en la articulación resecando todo el tejido fibroso. El extremo proximal del cúbito, en conjunto con el tendón del tríceps, se reparó hacia proximal (Figura 1B).

En todos los pacientes, el tríceps estaba contraído y sumamente adherido a la cara posterior del húmero, por lo que fue necesario liberarlo para permitir la posterior coaptación de los extremos del cúbito.

Los casos 1 y 2 eran secuelas de luxo-fracturas de Monteggia con una posterior evolución a una SO y degeneración articular. El caso 3 se trataba de una mujer con una fractura periprotésica por una artroplastia invertida de hombro que, un año antes, había presentado una fractura de olécranon tratada de manera conservadora y, en ese contexto, sufrió una fractura de húmero distal tipo C3 de la clasificación AO.

En los pacientes 1 y 2, se expuso todo el húmero distal y se realizaron los cortes correspondientes preservando ambas columnas. En el caso de la fractura supracondílea, se procedió a la resección del húmero distal fracturado.

A nivel cubital, se fresó el canal medular hasta permitir la colocación del implante correspondiente. A todos los pacientes, se les colocó una prótesis semiconstreñida de Coonard-Morrey (Zimmer, Warsaw, IN, EE.UU.) en dos casos y una Discovery (Biomet, Warsaw, IN, EE.UU.) en el restante.

A continuación, se colocó la prótesis de prueba ensamblada previamente. Este paso quirúrgico es importante, porque colocar el implante de esta manera evita la posibilidad de la mala rotación de los componentes, que es frecuente cuando hay un déficit óseo a nivel del cúbito proximal o del húmero distal. Para la colocación de la prótesis se ubicó el codo en máxima flexión y se introdujeron ambos componentes, en un mismo tiempo, en los canales medulares (Figura 1C). Luego se debe recortar el cúbito proximal para que se adapte mejor al cúbito distal.

Antes de cementar la prótesis se perforó un orificio transversal en el cúbito y se introdujo un alambre de 1,6 mm para el sistema de absorbe-tracción. Se colocó un tapón en el canal humeral y se cementó la prótesis definitiva con pistola. Siempre se utilizó cemento con antibiótico (vancomicina $1 \mathrm{~g} /$ dosis). Antes del fraguado del cemento, se redujo el fragmento de cúbito proximal y se colocaron dos clavijas de Kirschner de 1,6 mm desde la cara posterior del cúbito hasta la cara anterior o endomedular intentando colocarlas a cada lado del tallo de la prótesis. Las clavijas quedaron incluidas en el cemento para evitar su extrusión, y se colocó un injerto óseo en la zona de seudoartrosis obtenido de los cortes y fragmentos fracturarios del húmero distal (Figura 1D).

La osteosíntesis se realizó a $45^{\circ}$ de extensión del codo y se complementó con una sutura de hilo no reabsorbible (Ti-Cron ${ }^{\circledR} 2-0$ ) desde el tendón del tríceps hasta el orificio del sistema de absorbe-tracción con la intención de disminuir la tracción del tendón.

Durante la cirugía, se comprobó la movilidad completa del codo y se inmovilizó a los pacientes con una valva de yeso en $45^{\circ}$ de extensión, que se dejó por 15 días, luego se colocó un cabestrillo y se comenzó con un plan de movilización activa. 

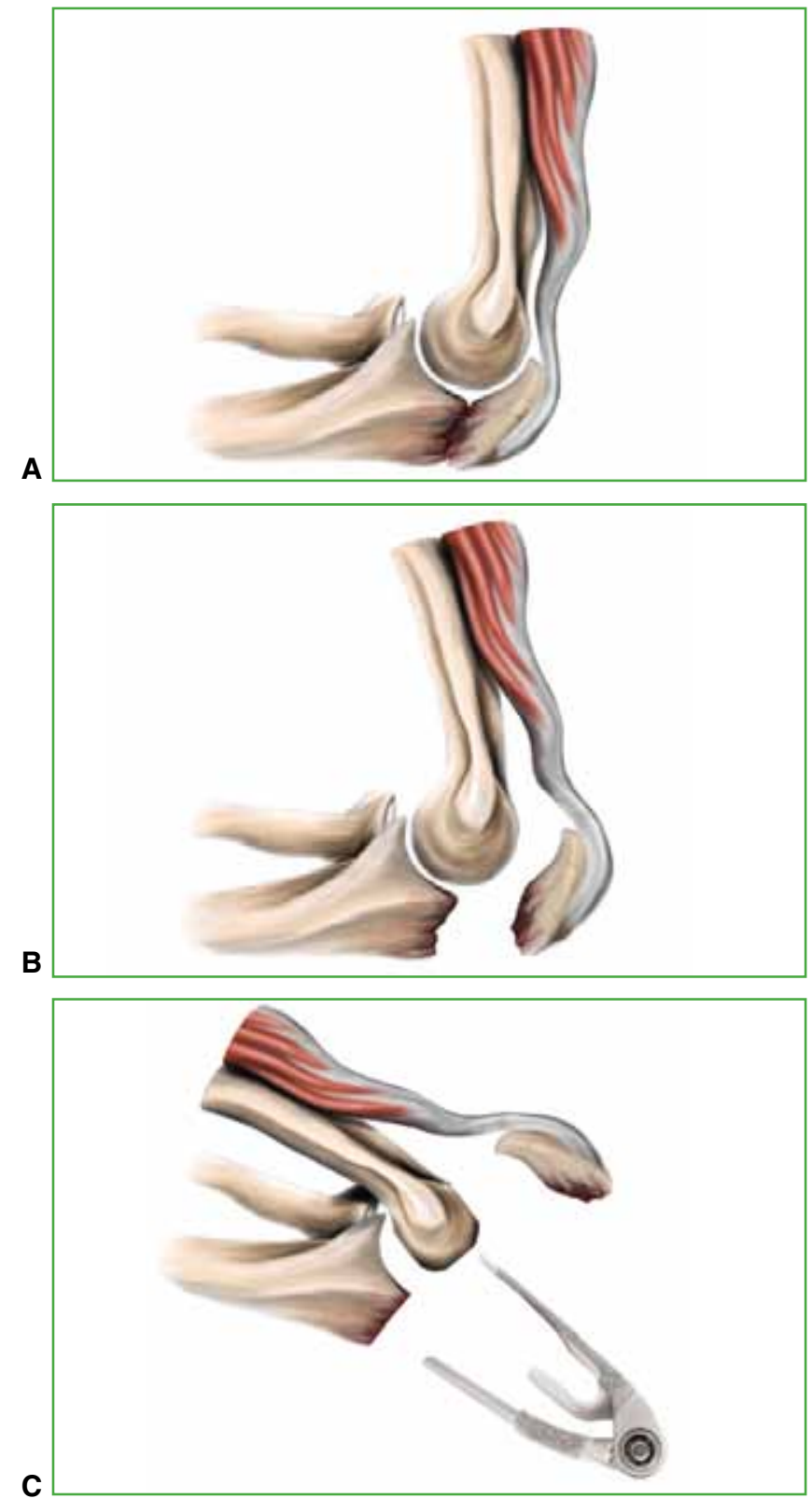

C

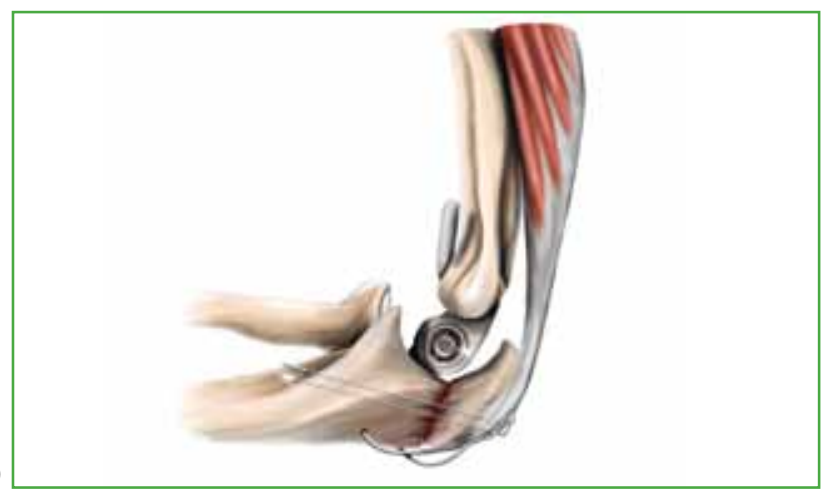

Figura 1. Técnica quirúrgica. A. Imagen con seudoartrosis de olécranon. B. Se ingresa a través del foco de seudoartrosis. C. Se realizan los cortes y se coloca la prótesis ensamblada. D. Se talla el olécranon proximal y se realiza la osteosíntesis con el sistema de absorbe-tracción. 


\section{CASOS CLÍNICOS}

Desde 2007 hasta la fecha, se colocaron 115 PTC en nuestro servicio. Solo tres PTC fueron colocadas en el contexto de una patología degenerativa, asociada a una SO.

\section{Caso 1}

Mujer de 70 años que consultó por dolor e inestabilidad en el codo izquierdo. Como antecedentes presentaba una luxo-fractura de Monteggia de tres años de evolución operada en tres oportunidades. En la primera cirugía, se le realizó la osteosíntesis del olécranon; en la segunda, la cupulectomía y, en la tercera, la extracción del material de osteosíntesis. La flexo-extensión era de $90^{\circ}-30^{\circ}$, con un puntaje de dolor de 8 en la escala analógica visual (EAV), un puntaje MEPS de 40 y un puntaje DASH de 56. En las radiografías, se observaba la SO y la avanzada degeneración articular. Previa punción para descartar infección la paciente fue operada con la técnica descrita. Se le colocó una prótesis de Coonrad-Morrey. A los cinco años de la cirugía, se le extrajeron las clavijas por molestias; la flexoextensión final fue de $125^{\circ}-25^{\circ}$, con un puntaje de 2 en la EAV, un puntaje MEPS de 75 y un puntaje DASH de 32. La seudoartrosis estaba consolidada y la prótesis no tenía signos de aflojamiento (Figura 2).

\section{Caso 2}

Hombre de 27 años que consultó por dolor e inestabilidad en el codo izquierdo. Como antecedentes presentaba una luxo-fractura de Monteggia de tres años de evolución, operada en cuatro oportunidades. En la primera cirugía, se le realizó la osteosíntesis del olécranon; luego, se le colocó un sistema de absorbe-tracción por subluxación, se resecó la cúpula radial y, por último, se extrajo toda la osteosíntesis. La flexo-extensión era de $110^{\circ}-40^{\circ}$, con un puntaje de dolor de 5 en la EAV, un puntaje MEPS de 35 y un puntaje DASH de 46. Las radiografías mostraban el desgaste articular y la SO. Previa punción para descartar infección, el paciente fue operado con la técnica descrita. Se le colocó una prótesis de Coonrad-Morrey. A los cuatro años de seguimiento, la flexo-extensión era de $120^{\circ}$ $35^{\circ}$, con un puntaje de 2 en la EAV, un puntaje MEPS de 75 y un puntaje DASH de 27. La seudoartrosis estaba consolidada y la prótesis no tenía signos de aflojamiento (Figura 3).

\section{Caso 3}

Mujer de 81 años, que consultó por una fractura supracondílea tipo C3 de la clasificación AO. Había sido operada por una fractura de hombro y se le había colocado una prótesis invertida. Luego sufrió una fractura periprotésica que fue tratada con una placa. Un año antes de nuestra cirugía, había tenido una fractura de olécranon tratada de manera incruenta. En nuestra cirugía, se extrajeron algunos tornillos distales del implante y se le colocó una prótesis Discovery. A los tres meses de la intervención, fue necesario retirar las clavijas por protrusión. Al año de seguimiento, la flexo-extensión era de $135^{\circ}-40^{\circ}$, con un puntaje de 2 en la EAV, puntaje MEPS de 80 y un puntaje DASH de 29. La seudoartrosis estaba consolidada y la prótesis no tenía signos de aflojamiento (Figura 4).

\section{DISCUSIÓN}

Las indicaciones más comunes del reemplazo protésico en el codo son secuelas postraumáticas y enfermedades degenerativas. La patología postraumática está asociada a un mayor número de complicaciones en comparación con las reumáticas. ${ }^{3,4}$ Dentro de ellas la insuficiencia del tríceps es una de las más publicadas ${ }^{9,14}$ y cuando el paciente tiene infecciones previas, los resultados son aún peores. Duquin y cols. ${ }^{15}$ comunicaron que el 55\% de 91 pacientes con antecedentes infecciosos en el codo tenía déficit de extensión del codo al final de la reconstrucción. Por lo tanto, el correcto y cuidadoso manejo del aparato extensor es fundamental para intentar prevenir las complicaciones.

Ante un cúbito proximal sin déficit óseo, se ha informado un relativo éxito de la reconstrucción con injertos de tendón de Aquiles de banco y colgajo rotatorio de ancóneo. Sanchez-Sotelo y Morrey reportaron siete pacientes con estos tipos de reconstrucción y una buena recuperación de la fuerza de extensión en seis de ellos. ${ }^{9}$

En casos con déficits óseos más severos, la solución es compleja. ${ }^{16-18}$

La SO altera la continuidad del aparato extensor y, por lo tanto, consideramos que debe solucionarse en el mismo momento de la colocación de la prótesis. La SO es infrecuente en el contexto de una fractura tratada con osteosíntesis. ${ }^{19,20}$

Papagelopoulos y Morrey ${ }^{12}$ publicaron una serie de 16 SO tratadas con injerto óseo autólogo y osteosíntesis; se logró la consolidación en 15 casos. Sin embargo, en luxo-fracturas de Monteggia o transolecraneanas, suele presentarse con mayor frecuencia. ${ }^{21,22}$ 

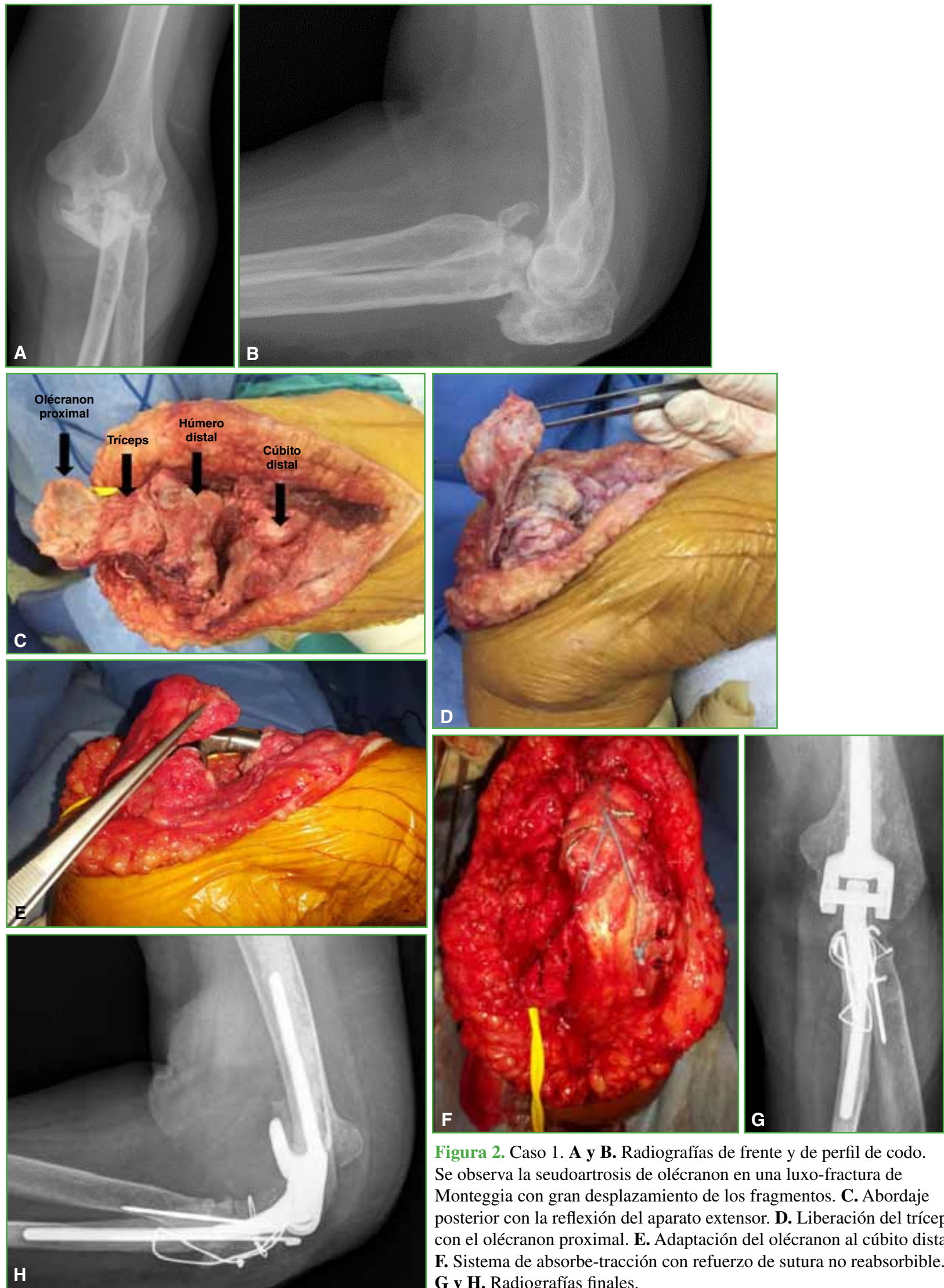

Figura 2. Caso 1. A y B. Radiografías de frente y de perfil de codo. Se observa la seudoartrosis de olécranon en una luxo-fractura de Monteggia con gran desplazamiento de los fragmentos. C. Abordaje posterior con la reflexión del aparato extensor. D. Liberación del tríceps con el olécranon proximal. E. Adaptación del olécranon al cúbito distal. F. Sistema de absorbe-tracción con refuerzo de sutura no reabsorbible. G y H. Radiografías finales. 

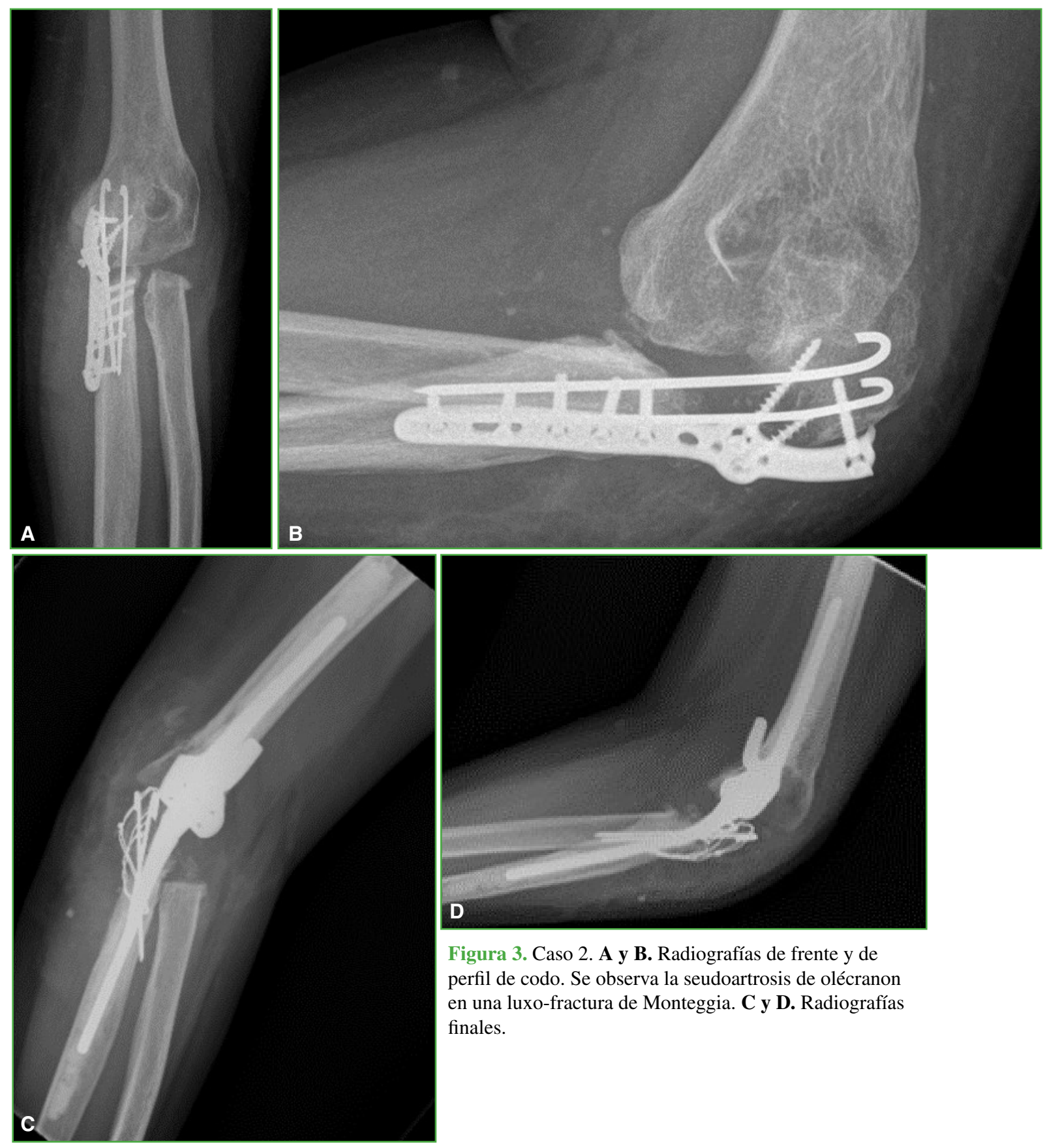

Figura 3. Caso 2. A y B. Radiografías de frente y de perfil de codo. Se observa la seudoartrosis de olécranon en una luxo-fractura de Monteggia. C y D. Radiografías finales. 


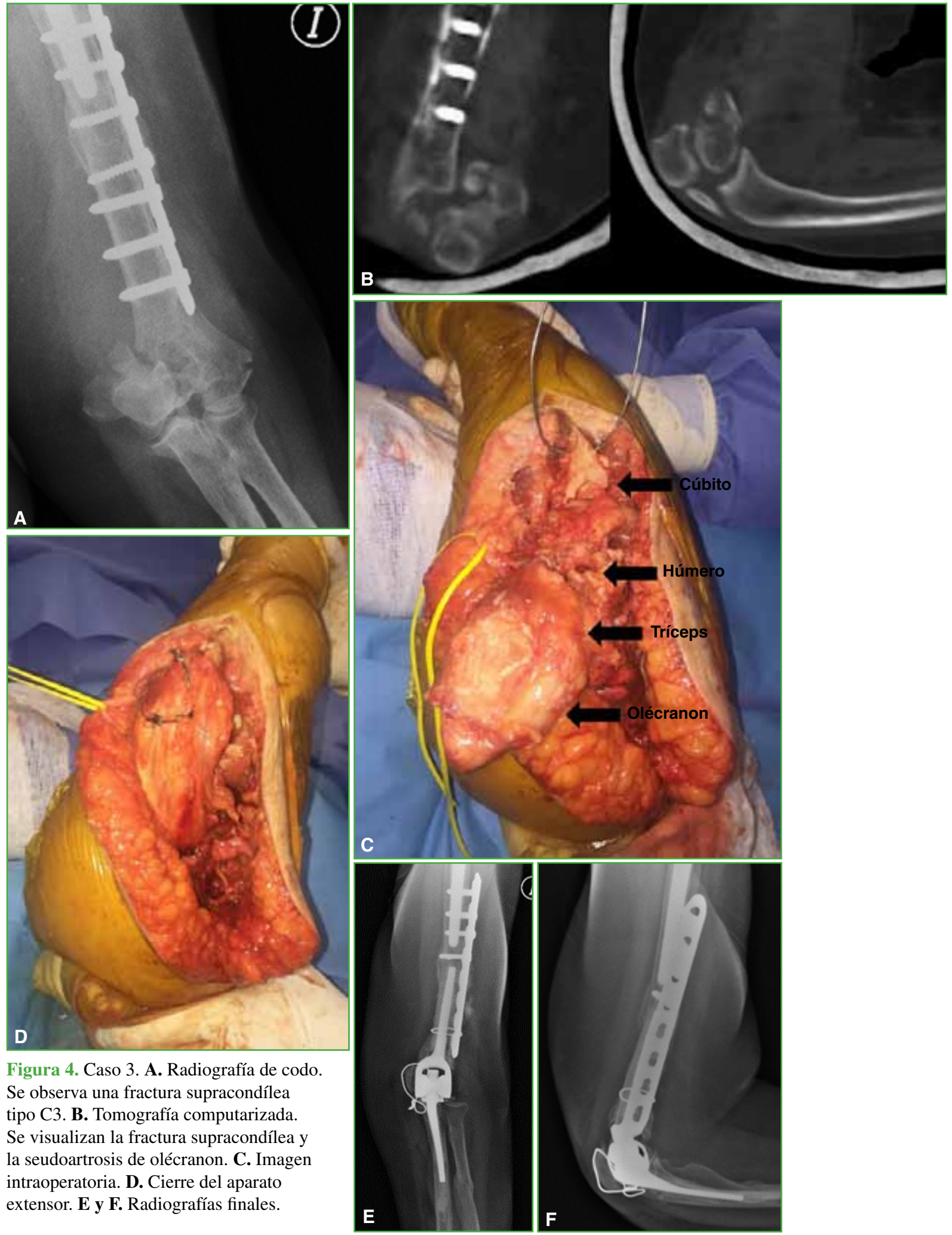


La técnica descrita permite colocar la prótesis manteniendo la continuidad del aparato extensor mediante su osteosíntesis con un sistema de absorbe-tracción.

Marra y cols. ${ }^{13}$ publicaron siete pacientes con patologías postraumáticas y SO a quienes les colocaron una PTC y, en cuatro de ellos, se logró la consolidación. Dos de los tres casos restantes sin consolidación requirieron una nueva intervención con osteosíntesis con placa y tornillos. Por lo tanto, la consolidación de la SO es difícil de obtener. En algunos casos, una fuerte unión fibrosa del foco seudoartrósico puede dar buenos resultados manteniendo una aceptable función del aparato extensor. ${ }^{13}$

En esta serie, hemos agregado injerto óseo tomado del húmero distal en los tres casos y se logró la consolidación en todos.

La retracción crónica del tríceps también puede favorecer la falta de consolidación. Por lo tanto, consideramos que el refuerzo con suturas no absorbibles y la inmovilización a $45^{\circ}$ de extensión son alternativas útiles para disminuir la fuerza de tracción muscular y permitir la consolidación de la seudoartrosis.

La colocación de las clavijas de Kirschner antes de que fragüe el cemento debe ser rápida y precisa, y con poca posibilidad de recolocación de estas. Por esta razón, debe medirse previamente el largo de las clavijas para que no sobrepasen la cortical anterior del cúbito. Sin embargo, hemos tenido que retirar las clavijas en dos de los casos por protrusión o molestias. La colocación del sistema de absorbe-tracción una vez fraguado el cemento en lugar de hacerlo con el cemento fresco, posiblemente sea un gesto útil para lograr un mejor posicionamiento de las clavijas, que ha sido un paso complicado en nuestros pacientes.

\section{CONCLUSIONES}

Cuando hay desgaste articular y una SO, el tratamiento con una PTC previamente ensamblada y colocada a través del foco de seudoartrosis, con liberación de todo el aparato extensor, una osteosíntesis con sistema de absorbe-tracción y el agregado de injerto óseo puede dar buenos resultados. Esta técnica nos permite lograr un codo estable, con poco dolor y mantener la continuidad del aparato extensor. Obtener resultados excelentes en este tipo de reconstrucción es difícil y el seguimiento de los pacientes es fundamental, porque los complicaciones son muy frecuentes.

Conflicto de intereses: Los autores no declaran conflictos de intereses.

\section{BIBLIOGRAFÍA}

1. Hackl M, Müller LP, Leschinger T, Wegmann K. Total elbow arthroplasty in traumatic and post-traumatic bone defects. Orthopade 2017;46(12):990-1000. https://doi.org/10.1007/s00132-017-3493-4

2. Kwak JM, Koh KH, Jeon IH. Total elbow arthroplasty: clinical outcomes, complications, and revision surgery. Clin Orthop Surg 2019;11(4):369-79. https://doi.org/10.4055/cios.2019.11.4.369

3. Wang JH, Ma HH, Chou TA, Tsai SW, Chen CF, Wu PK, et al. Outcomes following total elbow arthroplasty for rheumatoid arthritis versus post-traumatic conditions: a systematic review and meta-analysis. Bone Joint $\mathrm{J} \mathrm{Br}$ 2019;101(12):1489-97. https://doi-org/10.1302/0301-620X.101B12.BJJ-2019-0799.R1

4. Moro JK, King GJ. Total elbow arthroplasty in the treatment of posttraumatic conditions of the elbow. Clin Orthop Relat Res 2000;(370):102-14. https://doi.org/10.1097/00003086-200001000-00010

5. Toulemonde J, Ancelin D, Azoulay V, Bonnevialle N, Rongières M, Mansat P. Complications and revisions after semi-constrained total elbow arthroplasty: a mono-centre analysis of one hundred cases. Int Orthop 2016;40(1):7380. https://doi.org/10.1007/s00264-015-3008-Z 
6. Viveen J, van den Bekerom MPJ, Doornberg JN, Hatton A, Page R, Koenraadt KLM, et al. Use and outcome of 1,220 primary total elbow arthroplasties from the Australian Orthopaedic Association National Joint Arthroplasty Replacement Registry 2008-2018. Acta Orthop 2019;90(6):511-16. https://doi.org/10.1080/17453674.2019.1657342

7. Gallucci G, Warner Larrondo Calderón, Boretto J, Castellano Lanterno JA, Terán J, De Carli P. Artroplastia total de codo para el tratamiento de fracturas de húmero distal en pacientes mayores de 65 años. Rev Asoc Argent Ortop Traumatol 2016;81(2):84-92. https://doi.org/10.15417/521

8. Gallucci G, Gonzalez D, Boretto J, Alfie V, Donndorff A, De Carli P. Artroplastía total del codo. Rev Asoc Argent Ortop Traumatol 2010;75(1):27-33. Disponible en: https://www.aaot.org.ar/revista/2010/n1/art03.pdf

9. Sanchez-Sotelo J, Morrey BF. Surgical techniques for reconstruction of chronic insufficiency of the triceps. Rotation flap using anconeus and tendon achillis allograft. J Bone Joint Surg Br 2002;84(8):1116-20. https://doi.org/10.1302/0301-620x.84b8.12902

10. Morrey BF, Adams RA. Semiconstrained elbow replacement for distal humeral nonunion. J Bone Joint Surg Br 1995;77:67-72. PMID: 7822400

11. Morrey BF. Semiconstrained total elbow arthroplasty. En: Morrey BF (ed). The elbow. New York: Raven Press; 1994:231-55.

12. Papagelopoulos PJ, Morrey BF. Treatment of nonunion of olecranon fractures. J Bone Joint Surg Br 1994;76(4):627-35. PMID: 8027154

13. Marra G, Morrey BF, Gallay SH, McKee MD, O'Driscoll S. Fracture and nonunion of the olecranon in total elbow arthroplasty. J Shoulder Elbow Surg 2006;15:486-94. https://doi.org/10.1016/j.jse.2005.10.016

14. Morrey BF, Sanchez-Sotelo J. Approaches for elbow arthroplasty: how to handle the triceps. J Shoulder Elbow Surg 2011;20(2):S90-S96. https://doi.org/10.1016/j.jse.2010.12.004

15. Duquin TR, Jacobson JA, Schleck CD, Larson DR, Sanchez-Sotelo J, Morrey BF. Triceps insufficiency after the treatment of deep infection following total elbow replacement. Bone Joint J Br 2014;96(1):82-7. https://doi.org/10.1302/0301-620X.96B1.31127

16. Sanchez-Sotelo J, O’Driscoll S, Morrey BF. Periprosthetic humeral fractures after total elbow arthroplasty: treatment with implant revision and strut allograft augmentation. J Bone Joint Surg Am 2002;84:1642-50. PMID: 12208923

17. O’Driscoll SW, Morrey BF. Periprosthetic fractures about the elbow. Orthop Clin North Am 1999;30:319-25. https://doi.org/10.1016/s0030-5898(05)70086-9

18. Foruria AM, Sanchez-Sotelo J, Oh LS, Adams RA, Morrey BF. The surgical treatment of periprosthetic elbow fractures around the ulnar stem following semiconstrained total elbow arthroplasty. J Bone Joint Surg Am 2011;93(15):1399-1407. https://doi.org/10.2106/JBJS.J.00102

19. Danziger MB, Healy WL. Operative treatment of olecranon nonunion. J Orthop Trauma 1992;6:290-93. https://doi.org/10.1097/00005131-199209000-00004

20. Rotini R, Antonioli D, Marinelli A, Katusić D. Surgical treatment of proximal ulna nonunion. Chir Organi Mov 2008;91(2):65-70. https://doi.org/10.1007/s12306-007-0011-6

21. Wong JC, Getz CL, Abboud JA. Adult Monteggia and olecranon fracture dislocations of the elbow. Hand Clin 2015;31(4):565-80. https://doi.org/10.1016/j.hcl.2015.06.006

22. Mouhsine E, Akiki A, Castagna A, Cikes A, Wettstein M, Borens OR, et al. Transolecranon anterior fracture dislocation. J Shoulder Elbow Surg 2007;16(3):352-57. https://doi.org/10.1016/j.jse.2006.07.005 